\author{
Submitted to \\ manuscript (Please, provide the manuscript number!)
}

\title{
Semidefinite Relaxation for Two Mixed Binary Quadratically Constrained Quadratic Programs: Algorithms and Approximation Bounds
}

\author{
$\mathrm{Zi} \mathrm{Xu}$ \\ Department of Mathematics, College of Sciences, Shanghai University, Shanghai, 200444, P. R. China, xuzi@shu.edu.cn. \\ Mingyi Hong \\ Department of Electrical and Computer Engineering, University of Minnesota, Minneapolis, MN 55455, mhong@umn.edu.
}

This paper develops new semidefinite programming (SDP) relaxation techniques for two classes of mixed binary quadratically constrained quadratic programs (MBQCQP) and analyzes their approximation performance. The first class of problem finds two minimum norm vectors in $N$-dimensional real or complex Euclidean space, such that $M$ out of $2 M$ concave quadratic functions are satisfied. By employing a special randomized rounding procedure, we show that the ratio between the norm of the optimal solution of this model and its SDP relaxation is upper bounded by $\frac{54 M^{2}}{\pi}$ in the real case and by $\frac{24 M}{\sqrt{\pi}}$ in the complex case. The second class of problem finds a series of minimum norm vectors subject to a set of quadratic constraints and a cardinality constraint with both binary and continuous variables. We show that in this case the approximation ratio is also bounded and independent of problem dimension for both the real and the complex cases.

Key words: nonconvex quadratically constrained quadratic programming; semidefinite program relaxation; approximation bound; NP-hard

\section{Introduction}

In this paper, we study two classes of mixed binary nonconvex quadratically constrained quadratic programming (MBQCQP) problems, where the objective functions are quadratic in the continuous variables and the constraints contain both continuous and binary variables. These two classes of optimization problems are difficult as they are nonconvex even with the binary variables being fixed. The focus of our study is to design efficient semidefinite programming (SDP) based algorithms for both problems and to analyze their approximation bounds.

The first model. Consider the following MBQCQP problem:

$$
\begin{aligned}
\min _{\mathbf{w}_{1}, \mathbf{w}_{2} \in \mathbb{F}^{N}, \boldsymbol{\beta}} & \left\|\mathbf{w}_{1}\right\|^{2}+\left\|\mathbf{w}_{2}\right\|^{2} \\
\text { s.t. } & \mathbf{w}_{1}^{H} \mathbf{H}_{i} \mathbf{w}_{1} \geq \beta_{i}, i=1 \cdots, M, \\
& \mathbf{w}_{2}^{H} \mathbf{H}_{i} \mathbf{w}_{2} \geq 1-\beta_{i}, i=1 \cdots, M,
\end{aligned}
$$




$$
\beta_{i} \in\{0,1\}, i=1, \cdots, M
$$

where $\mathbb{F}$ is either the field of real numbers $\mathbb{R}$ or the field of complex numbers $\mathbb{C} ; \boldsymbol{\beta}:=\left(\beta_{1}, \cdots, \beta_{M}\right)^{T}$; $\mathbf{H}_{i}:=\mathbf{h}_{i} \mathbf{h}_{i}^{H}(i=1, \cdots, M)$ and $\mathbf{h}_{i}$ 's are $N$ dimensional real or complex vectors; $\|\cdot\|$ denotes the Euclidean norm in $\mathbb{F}^{N}$ and $M>0$ is a given integer. Throughout, we use the superscript $H$ to denote the complex Hermitian transpose when $\mathbb{F}=\mathbb{C}$, and use it to denote transpose if $\mathbb{F}=\mathbb{R}$. Notice that the problem ( $(\mathrm{P} 1)$ can be easily solved either when $N=1$ or $M=1$, by solving a maximum eigenvalue problem. Hence, we shall assume that $N \geq 2$ and $M \geq 2$ in the rest of the paper. Let $v^{\mathrm{P} 1}$ denote its optimal objective value. We note that problem (ㅍ1 $)$ is in general NPhard. The reason is that even if we fix all the binary variables, the problem is eqivalent to two subproblems, which are both NP-hard; see (Luo et al. 2007, Section 2).

Our interest in problem ( $(\mathrm{P} 1)$ is motivated by its application in telecommunications. For example, consider a cellular network where $M$ users, each equipped with a single receive antenna, are served by a base station (BS) with $N$ transmit antennas. Let $\overline{\mathbf{h}}_{i} \in \mathbb{C}^{N \times 1}$ denote the complex channel coefficient between the BS and user $i$, and let $n_{i}$ denote the thermal noise power at the receiver of user $i$. Let $\mathcal{M}=\{1, \cdots, M\}$ denote the set of all users. Assume that there are two time slots, and for each slot $q=\{1,2\}$ a linear transmit beam $\mathbf{w}_{q} \in \mathbb{C}^{N \times 1}$ is used by the BS to transmit a common message to the users. Using these notations, the signal to noise ratio (SNR) at the receiver of each user $i \in \mathcal{M}$ at a give slot can be expressed as $\mathrm{SNR}_{i} \triangleq \frac{\left|\mathbf{w}_{q}^{H} \overline{\mathbf{h}}_{i}\right|^{2}}{n_{i}}$. In order to successfully decode the transmitted message, typically a quality of service (QoS) requirement in the form of $\frac{\left|\mathbf{w}_{q}^{H} \overline{\mathbf{h}}_{i}\right|^{2}}{n_{i}} \geq \gamma_{i}$ is imposed by each user $i \in \mathcal{M}$, where $\gamma_{i}$ is a predetermined QoS threshold. Let us define $\mathbf{h}_{i}:=\frac{\overline{\mathbf{h}}_{i}}{\sqrt{n_{i} \gamma_{i}}}$ as user $i$ 's normalized channel.

When the number of users in the network is large, proper user scheduling algorithm is needed in order to guarantee the QoS for all the users. More specifically, the objective of the design is threefold: 1) Properly assign the users into different time slots; 2) Multicast the desired signal to each time slot; 3) Minimize the total transmit power across all slots. In the simplest case where there are only two slots available, the problem can be formulated as the MBQCQP probem (P1) by using $\beta_{i} \in\{0,1\}$ and $1-\beta_{i} \in\{0,1\}$ to represent whether user $i$ is scheduled in slot 1 or slot 2 . This problem is called joint user grouping and physical layer transmit beamforming.

In the special case where there is only a single slot available, the physical layer multicast problem tries to minimize the total transmit power while satisfying all the users' QoS constraint. Mathematically it is equivalent to problem (P1) with $\beta_{i}=1$ for all $i$. The resulting problem is a continuous QCQP problem, whose SDP relaxation bounds have been extensively studied; see e.g., Luo et al. (2007). When applying SDP relaxation to solve the related problem, the first step is to reformulate the problem by introducing a rank- 1 matrix $\mathbf{X}=\mathbf{w w}^{H}$. After dropping the nonconvex rank-1 constraint on $\mathbf{X}$, the relaxed problem becomes an SDP, whose optimal solution $\overline{\mathbf{X}}$ can be efficiently 
computed. A randomization procedure then follows which converts $\overline{\mathbf{X}}$ to a feasible solution. It has been shown in Luo et al. (2007) that such SDP relaxation scheme generates high-quality solutions, and the theoretical ratio between its optimal solution and the SDP relaxation has been shown to be upper bounded by $27 M^{2} / \pi$ Luo et al. (2007). However, for more general case (P1) with both binary and continuous variables, there is no known performance guarantees for the performance of SDP relaxation techniques.

The second model. Another interesting case of the MBQCQP problem is the generalization for the first model. In this more general model, we consider an alternative formulation which can deal with the case with multiple time slots. Consider the same system model as described in the first model, except that we have a total of $Q$ time slots available for scheduling. Let $\boldsymbol{\beta}:=\left[\boldsymbol{\beta}^{(1)}, \cdots, \boldsymbol{\beta}^{(Q)}\right]$, where $\boldsymbol{\beta}^{(q)} \in\{0,1\}^{M}$. Let $\mathbf{w}:=\left[\left(\mathbf{w}_{1}\right)^{H}, \cdots,\left(\mathbf{w}_{Q}\right)^{H}\right]^{H}$, where $\mathbf{w}_{q}$ represents the beamformer used in the $q$ th time slot. Consider the following problem

$$
\begin{aligned}
\min _{\mathbf{w}_{q}, \boldsymbol{\beta}^{(q)}} & \sum_{q=1}^{Q}\left\|\mathbf{w}_{q}\right\|^{2} \\
\text { s.t. } & \mathbf{w}_{q}^{H} \mathbf{H}_{i} \mathbf{w}_{q} \geq \beta_{i}^{(q)}, i=1 \cdots, M, q=1 \cdots, Q \\
& \sum_{q=1}^{Q} \beta_{i}^{(q)} \geq P_{i}, i=1, \cdots, M \\
& \beta_{i}^{(q)} \in\{0,1\}, \quad i=1, \cdots, M, q=1, \cdots Q .
\end{aligned}
$$

Note that we have generalized the problem to the one that allows each user $i$ to be assigned to at least $P_{i}$ time slots. Note that $P_{i} \in[1, \cdots, Q]$ is a given integer that can represent the users's service priorities. The higher the value of $P_{i}$, the larger the number of slots will be reserved for user $i$. let $V^{\mathrm{P} 2}$ denote its optimal objective value. Note that the above formulation, even for the $Q=2$ case, we have $M$ additional discrete integer variables compared with our previous formulation. For this more general case, no approximation bounds for SDP relaxation are known either.

In the absence of the discrete constraints, there is an extensive literature on the quality bounds of SDP relaxation for solving nonconvex QCQP problems, in either a maximization or a minimization form Luo et al. (2007), Nemirovski (1999), Ben-Tal et al. (2002), Beck and Teboulle (2009), He et al. (2008). In the seminal work by Goemans and Williamson (1995), the authors show that for the max-cut problem, which can be formulated as certain QCQP problem with discrete variables only, the ratio of the optimal value of SDP relaxation over that of the original problem is bounded below by $0.87856 \ldots$... Other related results can be found in Ye (2001), Frieze and Jerrum (1995). Some alternative approaches to MBQCQP have recently appeared in Billionnet et al. (2009), Burer and Saxena (2011), Saxena (2010), Saxena et al. (2011), Billionnet et al. (2012). 
More detailed reviews of recent progress on related problems can be found in the excellent surveys Burer and Letchford (2012), Hemmecke et al. (2010), Koppe (2011).

Although SDP relaxation technique has been quite successful in solving continuous QCQP, so far little is known about the effectiveness of applying it for MBQCQP, except for two recent results Hong et al. (2013), Xu et al. (2013). In those two papers, the authors study MBQCQP models that differ substantially from those considered in this work. In Hong et al. (2013), the authors considered a quadratic maximization problem with a single cardinality constraint on the binary variables, and those binary variables are used to reduce the dimensionality of continuous variables. In $\mathrm{Xu}$ et al. (2013), the authors consider an MBQCQP problem where the objective is to find a minimum norm vector under some concave mixed binary quadratic constraints and a single cardinality constraint on the binary variables. A special case of that problem is to find a minimum norm vector while satisfying $Q$ out of $M$ quadratic constraints. In this case the binary variables are used to reduce the number of quadratic constraints. In the current work, we consider a significantly harder problem where there are multiple cardinality constraints for the binary variables. As a result, the techniques and analysis developed in Hong et al. (2013), Xu et al. (2013) can not be applied directly. We note that if we set $M=1, P_{1}=q$ and $Q=m$, then problem $(\underline{\mathrm{P} 2})$ reduces to a special case of the minimization model considered in $\underline{\mathrm{Xu}}$ et al. (2013) with $\epsilon=0$.

Our Contributions. In Sections 2, we develop novel SDP relaxation techniques for solving the models (P1) and ( $(\mathrm{P} 2)$. The main idea is to first relax the binary variables to continuous variables and using the SDP relaxation for the rest of the continuous variables. Given an optimal solution of the relaxed problem, we devise new randomization procedures to generate approximate solutions for the original NP-hard MBQCQP problems. Moreover, we analyze the quality of such approximate solutions by deriving bounds on the approximation ratios between the optimal solution of the two MBQCQP problems and those of their corresponding SDP relaxations for both the real and complex cases.

Notations. For a symmetric matrix $\mathbf{X}, \mathbf{X} \succeq 0$ signifies that $\mathbf{X}$ is positive semi-definite. We use $\operatorname{Tr}[\mathbf{X}]$ and $\mathbf{X}[i, j]$ to denote the trace and the $(i, j)$ th element of a matrix $\mathbf{X}$, respectively. For a vector $\mathbf{x}$, we use $\|\mathbf{x}\|$ to denote its Euclidean norm, and use $\mathbf{x}[i]$ to denote its $i$ th element. For a real vector $\mathbf{y}$, use $\mathbf{y}_{[m]}$ to denote its $m$ th largest elements. For a complex scalar $x$, its complex conjugate is denoted by $\bar{x}$. Given a set $\mathcal{A},|\mathcal{A}|$ denotes the number of elements in set $\mathcal{A}$. Also, we use $\mathbb{R}^{N \times M}$ and $\mathbb{C}^{N \times M}$ to denote the set of real and complex $N \times M$ matrices, and use $\mathbb{S}^{N}$ and $\mathbb{S}_{+}^{N}$ to denote the set of $N \times N$ hermitian and hermitian positive semi-definite matrices, respectively. Finally, we use the superscript $H$ and $T$ to denote the complex Hermitian transpose and transpose of a matrix or a vector respectively. 


\section{Approximation Algorithm and Approximation Ratio Analysis}

2.1. The Proposed Algorithm for (P1) and Its Approximation Ratio

We consider a relaxation of the problem ( $(\overline{\mathrm{P} 1})$, expressed as follows

$$
\begin{aligned}
\min _{\mathbf{X}^{(1)}, \mathbf{X}^{(2)}, \boldsymbol{\alpha}} & \operatorname{Tr}\left[\mathbf{X}^{(1)}\right]+\operatorname{Tr}\left[\mathbf{X}^{(2)}\right] \\
\text { s.t. } & \operatorname{Tr}\left[\mathbf{H}_{i} \mathbf{X}^{(1)}\right] \geq \alpha_{i}, i=1, \cdots, M \\
& \operatorname{Tr}\left[\mathbf{H}_{i} \mathbf{X}^{(2)}\right] \geq 1-\alpha_{i}, i=1, \cdots, M \\
& 0 \leq \alpha_{i} \leq 1, i=1, \cdots, M \\
& \mathbf{X}^{(1)} \succeq 0, \mathbf{X}^{(2)} \succeq 0,
\end{aligned}
$$

where we do SDP relaxation for the continuous variables and continuous relaxation for the binary variables. Let $\left(\mathbf{X}^{*(1)}, \mathbf{X}^{*(2)}, \boldsymbol{\alpha}^{*}\right)$ denote the optimal solution to this SDP problem, and let $v^{\mathrm{SDP} 1}$ denote its optimal objective value.

In the following, we aim to generate a feasible solution $\left(\overline{\mathbf{x}}^{(1)}, \overline{\mathbf{x}}^{(2)}, \overline{\boldsymbol{\alpha}}^{*}\right)$ for (P1) from $\left(\mathbf{X}^{*(1)}, \mathbf{X}^{*(2)}, \boldsymbol{\alpha}^{*}\right)$, and evaluate the quality of such solution. In particular, we would like to find a constant $\mu \geq 1$ such that

$$
\left\|\overline{\mathbf{x}}^{(1)}\right\|^{2}+\left\|\overline{\mathbf{x}}^{(2)}\right\|^{2} \leq \mu v^{\mathrm{SDP} 1} .
$$

By using the fact that such generated solution is feasible for problem (표 $)$, we have $v^{\mathrm{P} 1} \leq\left\|\overline{\mathbf{x}}^{(1)}\right\|^{2}+$ $\left\|\overline{\mathbf{x}}^{(2)}\right\|^{2}$, which further implies that the same $\mu$ is an upper bound of the SDP relaxation performance, i.e.,

$$
v^{\mathrm{P} 1} \leq \mu v^{\mathrm{SDP} 1} .
$$

The constant $\mu$ will be referred to as the approximation ratio.

Once we obtain the solution $\left(\mathbf{X}^{*(1)}, \mathbf{X}^{*(2)}, \boldsymbol{\alpha}^{*}\right)$, we first need to convert it into a feasible solution for the problem ( $(\mathrm{P} 1)$. For such purpose, we propose a new randomization procedure; see Table 1.

\section{Table 1 The Randomization Procedure}

S0: Let $\overline{\boldsymbol{\alpha}}_{i}^{*}=1$ if $\boldsymbol{\alpha}_{i}^{*} \geq \frac{1}{2}$, otherwise let $\overline{\boldsymbol{\alpha}}_{i}^{*}=0$. Denote $\mathcal{I}=\left\{i \mid \overline{\boldsymbol{\alpha}}_{i}^{*}=1, i=1, \cdots, M\right\}$.

S1: Sample $\boldsymbol{\xi}^{(1)} \sim \mathcal{N}_{c}\left(\mathbf{0}, \mathbf{X}^{(\mathbf{1}) *}\right)$;

Sample $\boldsymbol{\xi}^{(2)} \sim \mathcal{N}_{c}\left(\mathbf{0}, \mathbf{X}^{(2) *}\right) ;$

S2: a) Let $t^{(1)}=\max _{i \in \mathcal{I}}\left\{\sqrt{\frac{1}{\boldsymbol{\xi}^{(1) H} H_{i} \boldsymbol{\xi}^{(1)}}}\right\}$;

b) Let $t^{(2)}=\max _{i \in \mathcal{M} \backslash \mathcal{I}}\left\{\sqrt{\frac{1}{\boldsymbol{\xi}^{(2) H} \mathbf{H}_{i} \boldsymbol{\xi}^{(2)}}}\right\}$;

S4: Let $\overline{\mathbf{x}}^{(1)}=t^{(1)} \boldsymbol{\xi}^{(1)}, \overline{\mathbf{x}}^{(2)}=t^{(2)} \boldsymbol{\xi}^{(2)}$. 
Note that after a single execution of the algorithm, we obtain a feasible solution $\left(\overline{\mathbf{x}}^{(1)}, \overline{\mathbf{x}}^{(2)}, \overline{\boldsymbol{\alpha}}^{*}\right)$ for the original problem ( $(\overline{\mathrm{P} 1})$, and we have

$$
v^{P 1}\left(\overline{\mathbf{x}}^{(1)}, \overline{\mathbf{x}}^{(2)}\right)=\left(t^{(1)}\right)^{2}\left\|\boldsymbol{\xi}^{(1)}\right\|^{2}+\left(t^{(2)}\right)^{2}\left\|\boldsymbol{\xi}^{(2)}\right\|^{2} .
$$

In the following two theorems, we will show the approximation ratio for our algorithm for both real and complex cases.

ThEOREM 1. There exists a constant $\sigma>0$ such that

$$
P\left(v^{\mathrm{P} 1}\left(\overline{\mathbf{x}}^{(1)}, \overline{\mathbf{x}}^{(2)}\right) \leq \mu v^{\mathrm{SDP} 1}\right) \geq \sigma,
$$

with $\mu=\frac{54 M^{2}}{\pi}$ when $\mathbb{F}=\mathbb{R}$ and $\mu=\frac{24 M}{\sqrt{\pi}}$ when $\mathbb{F}=\mathbb{C}$.

$L$ et us pick any $\alpha>0$, and we have the following series of inequalities

$$
\begin{aligned}
& \left(v^{\mathrm{P} 1}\left(\overline{\mathbf{x}}^{(1)}, \overline{\mathbf{x}}^{(2)}\right) \leq \mu v^{\mathrm{SDP} 1}\right) \\
= & P\left(\left\|\overline{\mathbf{x}}^{(1)}\right\|^{2}+\left\|\overline{\mathbf{x}}^{(2)}\right\|^{2} \leq \mu\left(\operatorname{Tr}\left[\mathbf{X}^{*(1)}\right]+\operatorname{Tr}\left[\mathbf{X}^{*(2)}\right]\right)\right) \\
\geq & P\left(\left\|\boldsymbol{\xi}^{(1)}\right\|^{2}+\left\|\boldsymbol{\xi}^{(2)}\right\|^{2} \leq \frac{\mu}{\alpha}\left(\operatorname{Tr}\left[\mathbf{X}^{*(1)}+\operatorname{Tr}\left[\mathbf{X}^{*(2)}\right)\right],\left(t^{(1)}\right)^{2} \leq \alpha,\left(t^{(2)}\right)^{2} \leq \alpha\right)\right. \\
\geq & 1-P\left(\left(t^{(1)}\right)^{2}>\alpha\right)-P\left(\left(t^{(2)}\right)^{2}>\alpha\right)-P\left(\left\|\boldsymbol{\xi}^{(1)}\right\|^{2}+\left\|\boldsymbol{\xi}^{(2)}\right\|^{2}>\frac{\mu}{\alpha}\left(\operatorname{Tr}\left[\mathbf{X}^{*(1)}+\operatorname{Tr}\left[\mathbf{X}^{*(2)}\right)\right]\right)\right. \\
= & P\left(\left(t^{(1)}\right)^{2} \leq \alpha\right)+P\left(\left(t^{(2)}\right)^{2} \leq \alpha\right)-1-P\left(\left\|\boldsymbol{\xi}^{(1)}\right\|^{2}+\left\|\boldsymbol{\xi}^{(2)}\right\|^{2}>\frac{\mu}{\alpha}\left(\operatorname{Tr}\left[\mathbf{X}^{*(1)}+\operatorname{Tr}\left[\mathbf{X}^{*(2)}\right)\right]\right)\right. \\
\geq & P\left(\frac{1}{\left(\boldsymbol{\xi}^{(1)}\right)^{H} \mathbf{H}_{i} \boldsymbol{\xi}^{(1)}} \leq \alpha, \forall i \in \mathcal{I}\right)+P\left(\frac{1}{\left(\boldsymbol{\xi}^{(2)}\right)^{H} \mathbf{H}_{i} \boldsymbol{\xi}^{(2)}} \leq \alpha, \forall i \in \mathcal{M} \backslash \mathcal{I}\right) \\
& -1-\frac{\alpha}{\mu} .
\end{aligned}
$$

where the last inequality is from the Markov's inequality. Since

$$
\begin{aligned}
& \operatorname{Tr}\left[\mathbf{H}_{i} \mathbf{X}^{*(1)}\right] \geq \alpha_{i}^{*} \geq \frac{1}{2}, \forall i \in \mathcal{I}, \\
& \operatorname{Tr}\left[\mathbf{H}_{i} \mathbf{X}^{*(2)}\right] \geq 1-\alpha_{i}^{*} \geq \frac{1}{2}, \forall i \in \mathcal{M} \backslash \mathcal{I},
\end{aligned}
$$

we have

$$
\begin{aligned}
P & \left(v^{\mathrm{P} 1}\left(\overline{\mathbf{x}}^{(1)}, \overline{\mathbf{x}}^{(2)}\right) \leq \mu v^{\mathrm{SDP} 1}\right) \\
\geq & P\left(\left(\boldsymbol{\xi}^{(1)}\right)^{H} \mathbf{H}_{i} \boldsymbol{\xi}^{(1)} \geq \frac{2 \operatorname{Tr}\left[\mathbf{H}_{i} \mathbf{X}^{*(1)}\right]}{\alpha}, \forall i \in \mathcal{I}\right) \\
& +P\left(\left(\boldsymbol{\xi}^{(2)}\right)^{H} \mathbf{H}_{i} \boldsymbol{\xi}^{(2)} \geq \frac{2 \operatorname{Tr}\left[\mathbf{H}_{i} \mathbf{X}^{*(2)}\right]}{\alpha}, \forall i \in \mathcal{M} \backslash \mathcal{I}\right)-1-\frac{\alpha}{\mu} \\
= & 1-P\left(\left(\boldsymbol{\xi}^{(1)}\right)^{H} \mathbf{H}_{i} \boldsymbol{\xi}^{(1)} \leq \frac{2 \operatorname{Tr}\left[\mathbf{H}_{i} \mathbf{X}^{*(1)}\right]}{\alpha}, \text { for some } i \in \mathcal{I}\right) \\
& -P\left(\left(\boldsymbol{\xi}^{(2)}\right)^{H} \mathbf{H}_{i} \boldsymbol{\xi}^{(2)} \leq \frac{2 \operatorname{Tr}\left[\mathbf{H}_{i} \mathbf{X}^{*(2)}\right]}{\alpha}, \text { for some } i \in \mathcal{M} \backslash \mathcal{I}\right)-\frac{\alpha}{\mu} .
\end{aligned}
$$


When $\mathbb{F}=\mathbb{R}$, then by utilizing (Luo et al. 2007, Lemma 1), and from (6), we obtain

$$
P\left(v^{\mathrm{P} 1}\left(\overline{\mathbf{x}}^{(1)}, \overline{\mathbf{x}}^{(2)}\right) \leq \mu v^{\mathrm{SDP} 1}\right) \geq 1-\sum_{i=1}^{M} \max \left\{\sqrt{\frac{2}{\alpha}}, \frac{2\left(\bar{r}_{i}-1\right)}{\pi-2} \cdot \frac{2}{\alpha}\right\}-\frac{\alpha}{\mu} .
$$

Since $\mathbf{H}_{i}=\mathbf{h}_{i} \mathbf{h}_{i}^{H}$, we have $\bar{r}_{i}=1$ for all $i=1, \cdots, M$ and thus

$$
P\left(v^{\mathrm{P} 1}\left(\overline{\mathbf{x}}^{(1)}, \overline{\mathbf{x}}^{(2)}\right) \leq \mu v^{\mathrm{SDP} 1}\right) \geq 1-M \cdot \sqrt{\frac{2}{\alpha}}-\frac{\alpha}{\mu} .
$$

By choosing $\alpha=\frac{18 M^{2}}{\pi}$, and set $\mu=3 \alpha$ and $\sigma=\frac{2-\sqrt{\pi}}{3}$, we have

$$
P\left(v^{\mathrm{P} 1}\left(\overline{\mathbf{x}}^{(1)}, \overline{\mathbf{x}}^{(2)}\right) \leq \mu v^{\mathrm{SDP} 1}\right) \geq \sigma>0
$$

When $\mathbb{F}=\mathbb{C}$, then by utilizing (Luo et al. 2007, Lemma 3), and from (6), we obtain

$$
P\left(v^{\mathrm{P} 1}\left(\overline{\mathbf{x}}^{(1)}, \overline{\mathbf{x}}^{(2)}\right) \leq \mu v^{\mathrm{SDP} 1}\right) \geq 1-\sum_{i=1}^{M} \max \left\{\frac{4}{3} \cdot \frac{2}{\alpha}, 16\left(\bar{r}_{i}-1\right)^{2} \cdot \frac{4}{\alpha^{2}}\right\}-\frac{\alpha}{\mu} .
$$

Since $\mathbf{H}_{i}=\mathbf{h}_{i} \mathbf{h}_{i}^{H}$, we have $\bar{r}_{i}=1$ for all $i=1, \cdots, M$ and thus

$$
P\left(v^{\mathrm{P} 1}\left(\overline{\mathbf{x}}^{(1)}, \overline{\mathbf{x}}^{(2)}\right) \leq \mu v^{\mathrm{SDP} 1}\right) \geq 1-M \cdot \frac{8}{3 \alpha}-\frac{\alpha}{\mu} .
$$

By choosing $\alpha=\frac{8 M}{\sqrt{\pi}}$, and set $\mu=3 \alpha$ and $\sigma=\frac{2-\sqrt{\pi}}{3}$, we have

$$
P\left(v^{\mathrm{P} 1}\left(\overline{\mathbf{x}}^{(1)}, \overline{\mathbf{x}}^{(2)}\right) \leq \mu v^{\mathrm{SDP} 1}\right) \geq \sigma>0
$$

This completes the proof.

TheOREM 2. For (IP1) and its SDP relaxation (SDP1), we have

$$
v^{\mathrm{P} 1} \leq \mu v^{\mathrm{SDP} 1},
$$

with $\mu=\frac{54 M^{2}}{\pi}$ when $\mathbb{F}=\mathbb{R}$ and $\mu=\frac{24 M}{\sqrt{\pi}}$ when $\mathbb{F}=\mathbb{C}$.

$W$ e see from the inequality (2) that there is a positive probability (independent of problem size) of at least

$$
\frac{2-\sqrt{\pi}}{3}=0.0758 \ldots
$$

that

$$
\max \left\{\max _{i \in \mathcal{I}}\left\{\sqrt{\frac{1}{\boldsymbol{\xi}^{(1) H} \mathbf{H}_{i} \boldsymbol{\xi}^{(1)}}}\right\}, \max _{i \in \mathcal{M} \backslash \mathcal{I}}\left\{\sqrt{\frac{1}{\boldsymbol{\xi}^{(2) H} \mathbf{H}_{i} \boldsymbol{\xi}^{(2)}}}\right\}\right\} \leq \alpha,
$$

with $\alpha=\frac{8 M}{\sqrt{\pi}}$ and

$$
\left\|\boldsymbol{\xi}^{(1)}\right\|^{2}+\left\|\boldsymbol{\xi}^{(2)}\right\|^{2} \leq 3\left(\operatorname{Tr}\left[\mathbf{X}^{*(1)}\right]+\operatorname{Tr}\left[\mathbf{X}^{*(2)}\right]\right) .
$$




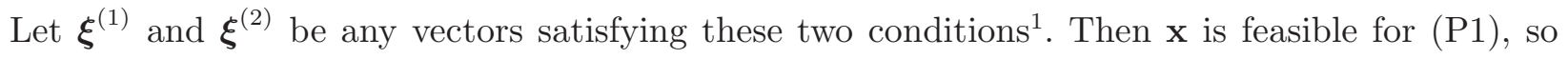
that

$$
\begin{aligned}
v^{\mathrm{P} 1} & \leq\left\|\overline{\mathbf{x}}^{(1)}\right\|^{2}+\left\|\overline{\mathbf{x}}^{(2)}\right\|^{2} \\
& =\left(\left\|\boldsymbol{\xi}^{(1)}\right\|^{2}+\left\|\boldsymbol{\xi}^{(2)}\right\|^{2}\right) \cdot \max \left\{\max _{i \in \mathcal{I}}\left\{\sqrt{\frac{1}{\boldsymbol{\xi}^{(1) H} \mathbf{H}_{i} \boldsymbol{\xi}^{(1)}}}\right\}, \max _{i \in \mathcal{M} \backslash \mathcal{I}}\left\{\sqrt{\frac{1}{\boldsymbol{\xi}^{(2) H} \mathbf{H}_{i} \boldsymbol{\xi}^{(2)}}}\right\}\right\} \\
& \leq \alpha \cdot 3\left(\operatorname{Tr}\left[\mathbf{X}^{*(1)}\right]+\operatorname{Tr}\left[\mathbf{X}^{*(2)}\right]\right)=\mu \cdot v^{\mathrm{SDP} 1},
\end{aligned}
$$

where the last equality uses $\operatorname{Tr}\left[\mathbf{X}^{*(1)}\right]+\operatorname{Tr}\left[\mathbf{X}^{*(2)}\right]=v^{\text {SDP1 }}$ and $\mu$ is defined as in Theorem 1 ,

\subsection{The Proposed Algorithm for ( $(\overline{\mathrm{P} 2})$ and Its Approximation Ratio}

We consider a relaxation of problem ( $(\overline{\mathrm{P} 2})$, expressed as follows:

$$
\begin{aligned}
\min _{\mathbf{\mathbf { X } ^ { ( q ) } , \boldsymbol { \alpha }}(q)} & \sum_{q=1}^{Q} \operatorname{Tr}\left[\mathbf{X}^{(q)}\right] \\
\text { s.t. } & \operatorname{Tr}\left[\mathbf{H}_{i} \mathbf{X}^{(q)}\right] \geq \alpha_{i}^{(q)}, i=1, \cdots, M, q=1 \cdots, Q, \\
& \sum_{q=1}^{Q} \alpha_{i}^{(q)} \geq P_{i}, i=1, \cdots, M, q=1 \cdots, Q, \\
& 0 \leq \alpha_{i}^{(q)} \leq 1, i=1, \cdots, M, q=1 \cdots, Q, \\
& \mathbf{X}^{(q)} \succeq 0, q=1 \cdots, Q,
\end{aligned}
$$

where we do SDP relaxation for the continuous variables and continuous relaxation for the binary variables. Let $\widehat{\mathbf{X}}^{(q)}$ and $\hat{\boldsymbol{\alpha}}^{(q)}=\left(\hat{\alpha}_{1}^{(q)}, \hat{\alpha}_{2}^{(q)}, \cdots, \hat{\alpha}_{M}^{(q)}\right)^{T}, q=1, \cdots, Q$ denote the optimal solution to this SDP problem, and let $v^{\mathrm{SDP} 2}=\sum_{q=1}^{Q} \operatorname{Tr}\left[\widehat{\mathbf{X}}^{(q)}\right]$ denote its optimal objective value.

We propose the algorithm listed in Table 2 to obtain a feasible solution $\left\{\left(\tilde{\mathbf{x}}^{(q)}, \tilde{\boldsymbol{\alpha}}^{(q)}\right)\right\}_{q=1, \cdots, Q}$ to the problem ( $(\overline{\mathrm{P} 2})$. The main idea of the algorithm is given below. For each user $i$, we first construct a set $\mathcal{P}_{i}$ that collects the largest $P_{i}$ components from $\left\{\hat{\alpha}_{i}^{(1)}, \cdots, \hat{\alpha}_{i}^{(q)}\right\}$. The binary variables are then determined using these sets $\mathcal{P}_{i}, i=1, \cdots, M$. Once the binary variables are fixed, standard randomization technique is use to construct each continuous variable $\tilde{\mathbf{x}}^{(q)}$.

Lemma 1. For all $i=1, \cdots, M$, if $q \in \mathcal{P}_{i}$, then $\hat{\alpha}_{i}^{(q)} \geq \frac{1}{Q-P_{i}+1}$.

$F \quad$ irst for any given $i$ in the set $\{1, \cdots, M\}$, by the feasibility of $\hat{\alpha}_{i}^{(q)}$, we have that

$$
\sum_{q=1}^{Q} \hat{\alpha}_{i}^{(q)} \geq P_{i}
$$

We claim that at least $P_{i}$ elements of the vector $\left\{\hat{\alpha}_{i}^{(1)}, \hat{\alpha}_{i}^{(2)}, \cdots, \hat{\alpha}_{i}^{(Q)}\right\}$ is greater than or equal to $\frac{1}{Q-P_{i}+1}$. We prove this claim by contradiction. Suppose that at most $P_{i}-1$ elements in this set is greater than or equal to $\frac{1}{Q-P_{i}+1}$. Then we have

$$
\sum_{q=1}^{Q} \hat{\alpha}_{i}^{(q)}<\left(Q-\left(P_{i}-1\right)\right)\left(\frac{1}{Q-P_{i}+1}\right)+\left(P_{i}-1\right)=P_{i} .
$$


S0: Denote $\mathcal{Q}:=\{1, \ldots, Q\}$ and define index sets:

$\mathcal{P}_{i}:=\left\{j \mid \hat{\alpha}_{i}^{(j)} \geq \hat{\alpha}_{i}^{\left[P_{i}\right]}, j=1, \cdots, Q\right\}, i=1, \cdots, M$,

where $\hat{\alpha}_{i}^{\left[P_{i}\right]}$ is the $P_{i}$-th largest element in vector $\left(\hat{\alpha}_{i}^{(1)}, \hat{\alpha}_{i}^{(2)}, \cdots, \hat{\alpha}_{i}^{(Q)}\right)$.

S1: For $i=1, \cdots, M$,

set $\tilde{\alpha}_{i}^{(q)}=1$ for all $q \in \mathcal{P}_{i}$ and $i=1, \cdots, M$;

otherwise set $\tilde{\alpha}_{i}^{(q)}=0$ for all $q \in \mathcal{Q} \backslash \mathcal{P}_{i}$ and $i=1, \cdots, M$.

Let $\tilde{\boldsymbol{\alpha}}^{(q)}=\left(\tilde{\alpha}_{1}^{(q)}, \tilde{\alpha}_{2}^{(q)}, \cdots, \tilde{\alpha}_{M}^{(q)}\right)^{T}$ for all $q=1, \cdots, Q$.

S2: For all $q \in \mathcal{Q}$, denote

$\mathcal{S}^{(q)}=\left\{i \mid \tilde{\alpha}_{i}^{(q)}=1, i=1, \cdots, M\right\}$.

S3: Generate a random vector $\boldsymbol{\xi}^{(q)}$ from the Normal distribution $\mathcal{N}_{c}\left(\mathbf{0}, \widehat{\mathbf{X}}^{(q)}\right), q=1, \cdots, Q$.

S4: Let $\tilde{\mathbf{x}}^{(q)}=\tilde{t}^{(q)} \boldsymbol{\xi}^{(q)}$, with

$\tilde{t}^{(q)}=\sqrt{\max _{i \in \mathcal{S}^{(q)}}\left\{\frac{1}{\boldsymbol{\xi}^{(q) H} \mathbf{H}_{i} \boldsymbol{\xi}^{(q)}}\right\}}$, for all $q=1, \cdots, Q$.

This contradicts (13). The claim is proved. Since $\mathcal{P}_{i}$ contains the $P_{i}$ largest elements of the vector $\left\{\hat{\alpha}_{i}^{(1)}, \hat{\alpha}_{i}^{(2)}, \cdots, \hat{\alpha}_{i}^{(Q)}\right\}$, this completes the proof.

Next, we analyze the above proposed algorithm.

THEOREM 3. There exists a positive constant $\sigma_{2}$ such that

$$
\operatorname{Prob}\left(\sum_{q=1}^{Q}\left\|\tilde{\mathbf{x}}^{q}\right\|^{2} \leq \bar{\mu}\left(\sum_{q=1}^{Q} \operatorname{Tr}\left[\widehat{\mathbf{X}}^{(q)}\right]\right)\right) \geq \sigma_{2}>0 .
$$

with

$$
\bar{\mu}=\frac{27\left(\sum_{i=1}^{M} P_{i} \sqrt{Q-P_{i}+1}\right)^{2}}{\pi}, \text { when } \mathbb{F}=\mathbb{R}
$$

and

$$
\bar{\mu}=\frac{12 \sum_{i=1}^{M} P_{i}\left(Q-P_{i}+1\right)}{\sqrt{\pi}}, \text { when } \mathbb{F}=\mathbb{C} .
$$

$T$ he desired probability can be bounded below as follows

$$
\begin{aligned}
& \operatorname{Prob}\left(v^{\mathrm{P} 2} \leq \bar{\mu} v^{\mathrm{SDP} 2}\right) \\
\geq & \operatorname{Prob}\left(\sum_{q=1}^{Q}\left\|\tilde{\mathbf{x}}^{q}\right\|^{2} \leq \bar{\mu}\left(\sum_{q=1}^{Q} \operatorname{Tr}\left[\widehat{\mathbf{X}}^{(q)}\right]\right)\right) \\
\geq & \operatorname{Prob}\left(\sum_{q=1}^{Q}\left(\tilde{t}^{(q)}\right)^{2}\left\|\boldsymbol{\xi}^{(q)}\right\|^{2} \leq \bar{\mu}\left(\sum_{q=1}^{Q} \operatorname{Tr}\left[\widehat{\mathbf{X}}^{(q)}\right]\right)\right) \\
\geq & \operatorname{Prob}\left(\sum_{q=1}^{Q}\left\|\boldsymbol{\xi}^{(q)}\right\|^{2} \leq \frac{\bar{\mu}}{\alpha_{2}}\left(\sum_{q=1}^{Q} \operatorname{Tr}\left[\widehat{\mathbf{X}}^{(q)}\right]\right), \frac{1}{\boldsymbol{\xi}^{(q)} \mathbf{H}_{i} \boldsymbol{\xi}^{(q)}} \leq \alpha_{2}, \forall i \in \mathcal{S}^{(q)} \text { and } q \in \mathcal{Q}\right) .
\end{aligned}
$$

Now notice that for all $i \in \mathcal{M}$ and $q \in \mathcal{Q}$, if $i \in \mathcal{S}^{(q)}$, then it can be easily checked that $q \in \mathcal{P}_{i}$. It follows that, by using Lemma 1 we have that

$$
\operatorname{Tr}\left[\mathbf{H}_{i} \hat{\mathbf{X}}^{(q)}\right] \geq \hat{\alpha}_{i}^{(q)} \geq \frac{1}{Q-P_{i}+1}, \text { when } i \in \mathcal{S}^{(q)} \text { and } q \in \mathcal{Q} \text {. }
$$


Then, we have

$$
\begin{aligned}
& \operatorname{Prob}\left(v^{\mathrm{P} 2} \leq \bar{\mu} v^{\mathrm{SDP} 2}\right) \\
\geq \operatorname{Prob}\left[\sum_{q=1}^{Q}\left\|\boldsymbol{\xi}^{(q)}\right\|^{2} \leq \frac{\bar{\mu}}{\alpha_{2}}\left(\sum_{q=1}^{Q} \operatorname{Tr}\left[\widehat{\mathbf{X}}^{(q)}\right]\right)\right. & \left.\boldsymbol{\xi}^{(q)} \mathbf{H}_{i} \boldsymbol{\xi}^{(q)} \geq \frac{Q-P_{i}+1}{\alpha_{2}} \operatorname{Tr}\left[\mathbf{H}_{i} \hat{\mathbf{X}}^{(q)}\right], \forall i \in \mathcal{S}^{(q)} \text { and } q \in \mathcal{Q}\right] \\
\geq & 1-\operatorname{Prob}\left(\sum_{q=1}^{Q}\left\|\boldsymbol{\xi}^{(q)}\right\|^{2}>\frac{\bar{\mu}}{\alpha_{2}}\left(\sum_{q=1}^{Q} \operatorname{Tr}\left[\widehat{\mathbf{X}}^{(q)}\right]\right)\right) \\
& -\sum_{q=1}^{Q} \sum_{i \in \mathcal{S}^{(q)}} \operatorname{Prob}\left(\boldsymbol{\xi}^{(q) H} \mathbf{H}_{i} \boldsymbol{\xi}^{(q)}<\frac{Q-P_{i}+1}{\alpha_{2}} \operatorname{Tr}\left[\mathbf{H}_{i} \hat{\mathbf{X}}^{(q)}\right]\right) \\
= & -\operatorname{Prob}\left(\sum_{q=1}^{Q}\left\|\boldsymbol{\xi}^{(q)}\right\|^{2}>\frac{\bar{\mu}}{\alpha_{2}}\left(\sum_{q=1}^{Q} \operatorname{Tr}\left[\widehat{\mathbf{X}}^{(q)}\right]\right)\right) \\
& -\sum_{i=1}^{M} \sum_{q \in \mathcal{P}_{i}} \operatorname{Prob}\left(\boldsymbol{\xi}^{(q) H} \mathbf{H}_{i} \boldsymbol{\xi}^{(q)}<\frac{Q-P_{i}+1}{\alpha_{2}} \operatorname{Tr}\left[\mathbf{H}_{i} \hat{\mathbf{X}}^{(q)}\right]\right) \\
\geq 1- & \frac{\alpha_{2}}{\bar{\mu}}-\sum_{i=1}^{M} \sum_{q \in \mathcal{P}_{i}} \operatorname{Prob}\left(\boldsymbol{\xi}^{(q) H} \mathbf{H}_{i} \boldsymbol{\xi}^{(q)}<\frac{Q-P_{i}+1}{\alpha_{2}} \operatorname{Tr}\left[\mathbf{H}_{i} \hat{\mathbf{X}}^{(q)}\right]\right),
\end{aligned}
$$

where the last inequality is from the Markov's inequality.

The Real Case. When $\mathbb{F}=\mathbb{R}$, by (Luo et al. 2007, Lemma 1), we have that

$$
\operatorname{Prob}\left(v^{\mathrm{P} 2} \leq \bar{\mu} v^{\mathrm{SDP} 2}\right) \geq 1-\frac{\alpha_{2}}{\bar{\mu}}-\sum_{i=1}^{M} P_{i} \cdot \delta_{i}\left(\alpha_{2}\right),
$$

with

$$
\delta_{i}\left(\alpha_{2}\right)=\max \left\{\sqrt{\frac{Q-P_{i}+1}{\alpha_{2}}}, \frac{2\left(r_{i}-1\right)}{\pi-2} \cdot \frac{Q-P_{i}+1}{\alpha_{2}}\right\},
$$

and $r_{i}=\min \left\{\operatorname{Rank}\left(\mathbf{H}_{i}\right), \operatorname{Rank}\left(\hat{\mathbf{X}}^{(q)}\right)\right\}$. Since $\mathbf{H}_{i}=\mathbf{h}_{i} \mathbf{h}_{i}^{H}$, we have $\bar{r}_{i}=1$ for all $i=1, \cdots, M$ and thus $\delta_{i}\left(\alpha_{2}\right)=\sqrt{\frac{Q-P_{i}+1}{\alpha_{2}}}$. As a result, we have

$$
\operatorname{Prob}\left(v^{\mathrm{P} 2} \leq \bar{\mu} v^{\mathrm{SDP} 2}\right) \geq 1-\frac{\alpha_{2}}{\bar{\mu}}-\sum_{i=1}^{M} P_{i} \cdot \sqrt{\frac{Q-P_{i}+1}{\alpha_{2}}} .
$$

By setting $\alpha_{2}=\frac{9\left(\sum_{i=1}^{M} P_{i} \sqrt{Q-P_{i}+1}\right)^{2}}{\pi}, \bar{\mu}=3 \alpha_{2}$, and $\sigma_{2}=\frac{2-\sqrt{\pi}}{3}$, we have

$$
\operatorname{Prob}\left(v^{\mathrm{P} 2} \leq \bar{\mu} v^{\mathrm{SDP} 2}\right) \geq \sigma_{2}>0
$$

The Complex Case. When $\mathbb{F}=\mathbb{C}$, by (Luo et al. 2007, Lemma 3), we have that

$$
\operatorname{Prob}\left(v^{\mathrm{P} 2} \leq \bar{\mu} v^{\mathrm{SDP} 2}\right) \geq 1-\frac{\alpha_{2}}{\bar{\mu}}-\sum_{i=1}^{M} P_{i} \cdot \bar{\delta}_{i}\left(\alpha_{2}\right),
$$


with

$$
\bar{\delta}_{i}\left(\alpha_{2}\right)=\max \left\{\frac{4}{3} \cdot \frac{Q-P_{i}+1}{\alpha_{2}}, 16\left(r_{i}-1\right)^{2} \cdot \frac{\left(Q-P_{i}+1\right)^{2}}{\alpha_{2}^{2}}\right\},
$$

and $r_{i}=\min \left\{\operatorname{Rank}\left(\mathbf{H}_{i}\right), \operatorname{Rank}\left(\hat{\mathbf{X}}^{(q)}\right)\right\}$. Since $\mathbf{H}_{i}=\mathbf{h}_{i} \mathbf{h}_{i}^{H}$, we have $\bar{r}_{i}=1$ for all $i=1, \cdots, M$ and thus $\bar{\delta}_{i}\left(\alpha_{2}\right)=\frac{4}{3} \cdot \frac{Q-P_{i}+1}{\alpha_{2}}$. As a result, we have

$$
\operatorname{Prob}\left(v^{\mathrm{P} 2} \leq \bar{\mu} v^{\mathrm{SDP} 2}\right) \geq 1-\frac{\alpha_{2}}{\bar{\mu}}-\frac{4}{3} \sum_{i=1}^{M} P_{i} \cdot \frac{Q-P_{i}+1}{\alpha_{2}} .
$$

By setting $\alpha_{2}=\frac{4 \sum_{i=1}^{M} P_{i}\left(Q-P_{i}+1\right)}{\sqrt{\pi}}, \bar{\mu}=3 \alpha_{2}$, and $\sigma_{2}=\frac{2-\sqrt{\pi}}{3}$, we have

$$
\operatorname{Prob}\left(v^{\mathrm{P} 2} \leq \bar{\mu} v^{\mathrm{SDP} 2}\right) \geq \sigma_{2}>0
$$

This completes the proof.

TheOREM 4. For (IP2) and its SDP relaxation (SDP2), we have

$$
v^{\mathrm{P} 2} \leq \bar{\mu} v^{\mathrm{SDP} 2},
$$

with $\bar{\mu}=\frac{27\left(\sum_{i=1}^{M} P_{i} \sqrt{Q-P_{i}+1}\right)^{2}}{\pi}$ when $\mathbb{F}=\mathbb{R}$ and $\bar{\mu}=\frac{12 \sum_{i=1}^{M} P_{i}\left(Q-P_{i}+1\right)}{\sqrt{\pi}}$ when $\mathbb{F}=\mathbb{C}$.

The proof of Theorem 4 is similar to that of Theorem 2, and we omit it.

It is important to mention here that, $P_{i}=1$ for all $i$ and $Q=1$ in the real case, we have

$$
\bar{\mu}=\frac{27 M^{2}}{\pi},
$$

which corresponding to Luo et al.'s result Luo et al. (2007). Moreover, when we consider case that $Q=2$ and $P_{i}=1$ for all $i$, the proposed algorithm and the approximation analysis reduces to the one that we have discussed in Section 2.1. Furthermore, if we set $M=1, P_{1}=q$ and $Q=m$ in the real case, we have

$$
\bar{\mu}=\frac{27 q^{2}(m-q+1)}{\pi},
$$

which is exactly the results that we have obtained for the minimization model with $\epsilon=0$ as in Xu et al. (2013).

\section{Numerical Experiments}

In this section we perform numerical study for the proposed algorithms. Throughout this section, we generate the data matrix $\mathbf{H}_{i}$ by using $\mathbf{H}_{i}=\mathbf{h}_{i} \mathbf{h}_{i}^{H}(i=1, \cdots, M)$, with randomly generated vectors $\mathbf{h}_{i}$. The SDP relaxation problems are all solved by CVX Grant and Boyd (2011), and the optimal objective value for the SDPs are denoted by $v_{\mathrm{SDP}}^{\min }$. 
Figure 1 Upper bound on $v_{\mathrm{QP}}^{\min } / v_{\mathrm{SDP}}^{\min }$ for $M=5, N=4,300$ realizations of real Gaussian i.i.d. $\mathbf{h}_{i}$ for $i=1, \cdots, M$ in the real case.

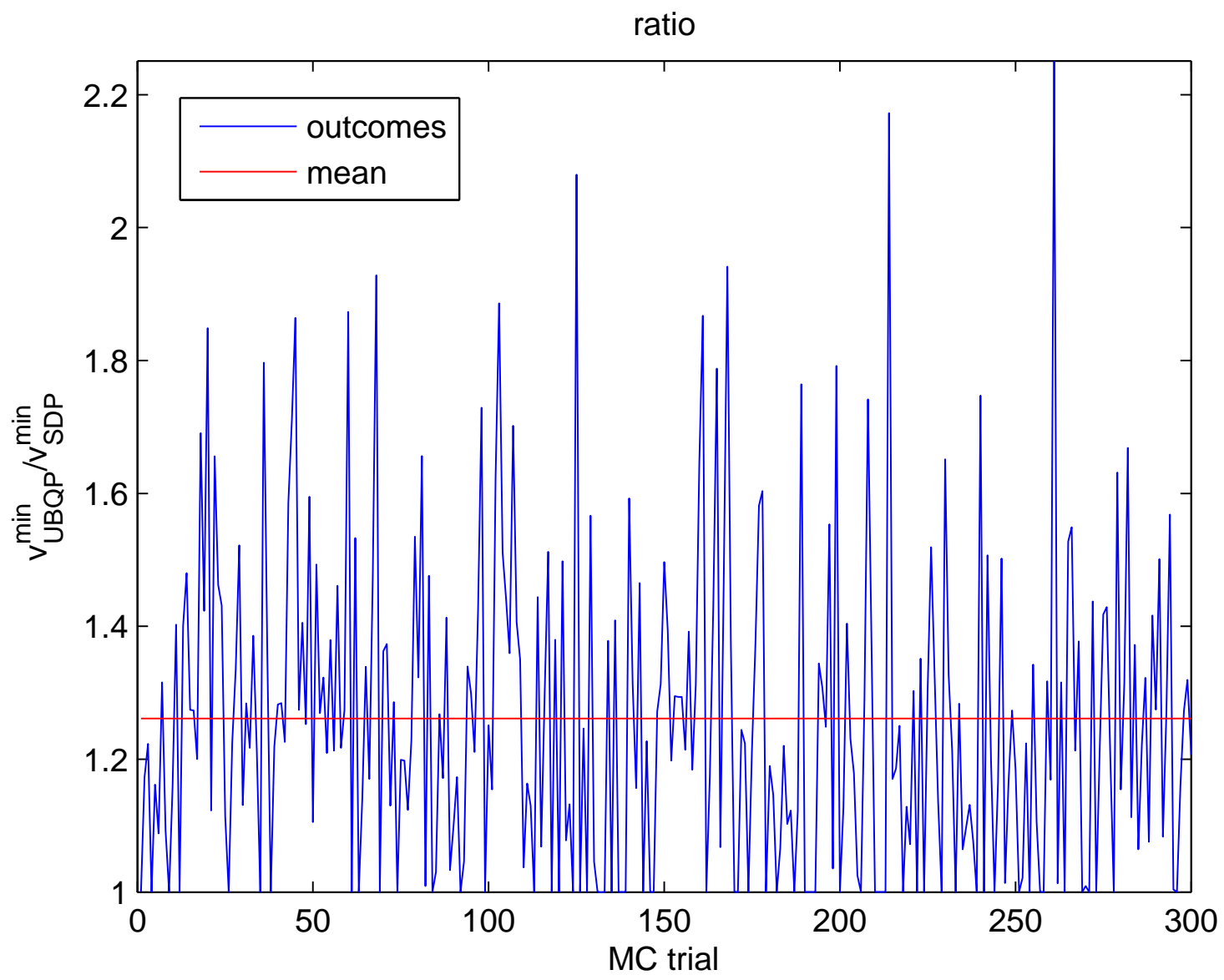

Table 3 Mean and standard deviation of the approximation ratio over 300 independent realizations of real Gaussian i.i.d. $\mathbf{h}_{i}(i=1, \cdots, M)$, when $\mathbb{F}=\mathbb{R}$.

\begin{tabular}{|l|l|cccc|}
\hline$M$ & $N$ & $\min$ & $\max$ & mean & Std \\
\hline \multirow{3}{*}{$M=5$} & $N=4$ & 1.0000 & 2.2508 & 1.2612 & 0.0590 \\
& $N=6$ & 1.0000 & 4.1582 & 1.2938 & 0.1467 \\
& $N=8$ & 1.0000 & 2.4780 & 1.3045 & 0.0985 \\
\hline \multirow{3}{*}{$M=10$} & $N=4$ & 1.0000 & 5.5210 & 1.7504 & 0.4253 \\
& $N=6$ & 1.0000 & 5.3879 & 1.7640 & 0.3313 \\
& $N=8$ & 1.0000 & 6.9469 & 2.1074 & 0.8202 \\
\hline \multirow{3}{*}{$M=15$} & $N=4$ & 1.0000 & 9.2963 & 2.2168 & 1.0545 \\
& $N=6$ & 1.0000 & 6.7141 & 2.6144 & 1.1682 \\
& $N=8$ & 1.3109 & 6.5277 & 2.9619 & 1.1806 \\
\hline
\end{tabular}

We test the proposed procedure listed in Table 1 for (P1) with different choices of $M$ and $N$. The Step S3 and Step S4 are repeated by $T=1000$ independent trials, and the solutions generated 
Figure 2 Histogram of the outcomes in Figure 1 .

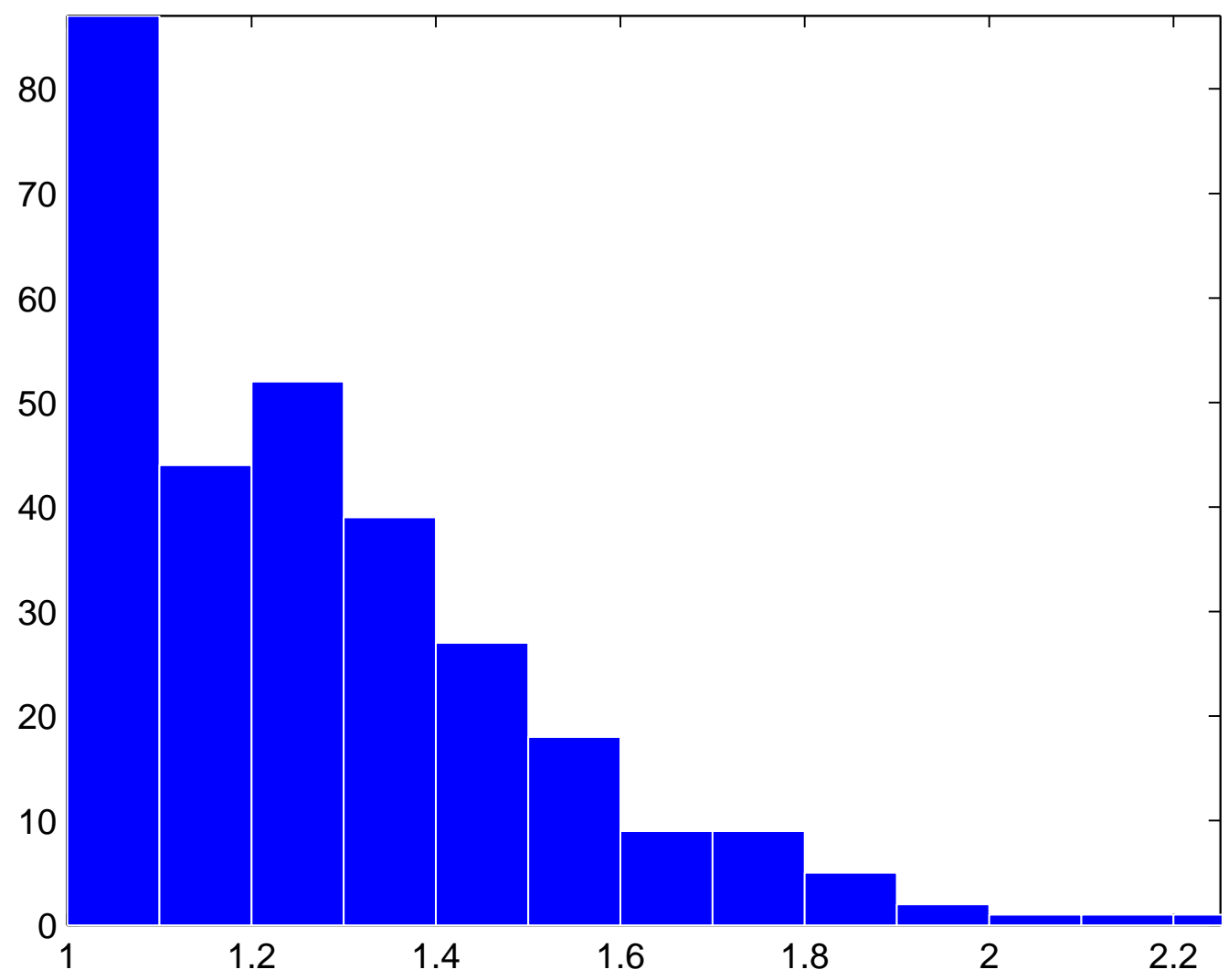

Table 4 Mean and standard deviation of upper bound ratio over 300 independent realizations of real Gaussian i.i.d. $\mathbf{h}_{i}(i=1, \cdots, M)$, when $\mathbb{F}=\mathbb{C}$.

\begin{tabular}{|l|l|cccc|}
\hline$M$ & $N$ & $\min$ & $\max$ & $\operatorname{mean}$ & Std \\
\hline \multirow{3}{*}{$M=5$} & $N=4$ & 1.0000 & 1.8518 & 1.1336 & 0.0445 \\
& $N=6$ & 1.0000 & 1.9089 & 1.0870 & 0.0341 \\
& $N=8$ & 1.0000 & 1.9620 & 1.0915 & 0.0400 \\
\hline \multirow{3}{*}{$M=10$} & $N=4$ & 1.0000 & 2.2457 & 1.5281 & 0.0654 \\
& $N=6$ & 1.0000 & 2.2511 & 1.5912 & 0.0781 \\
& $N=8$ & 1.0000 & 2.3561 & 1.5885 & 0.0828 \\
\hline \multirow{3}{*}{$M=15$} & $N=4$ & 1.0097 & 2.8260 & 1.7460 & 0.0699 \\
& $N=6$ & 1.0001 & 3.0243 & 1.8821 & 0.1064 \\
& $N=8$ & 1.0741 & 3.4917 & 1.9442 & 0.1193 \\
\hline
\end{tabular}

by $k$ th trial are denoted by $\left(\overline{\mathbf{x}}^{(1)}\right)^{k}$ and $\left(\overline{\mathbf{x}}^{(2)}\right)^{k}$. Let

$$
v_{\mathrm{UBQP}}^{\min }:=\min _{k=1, \cdots, T}\left\|\left(\overline{\mathbf{x}}^{(1)}\right)^{k}\right\|^{2}+\left\|\left(\overline{\mathbf{x}}^{(2)}\right)^{k}\right\|^{2}
$$

It is clearly that $v_{\mathrm{UBQP}}^{\min } \geq v^{\mathrm{P} 1}$, as a result, $v_{\mathrm{UBQP}}^{\min } / v_{\mathrm{SDP}}^{\min }$ is an upper bound of the true approximation ratio (which is difficult to obtain in polynomial time). 
Figure 3 Upper bound on $v_{\mathrm{QP}}^{\min } / v_{\mathrm{SDP}}^{\min }$ for $M=5, N=4,300$ realizations of real Gaussian i.i.d. $\mathbf{h}_{i}$ for $i=1, \cdots, M$ in the complex case.

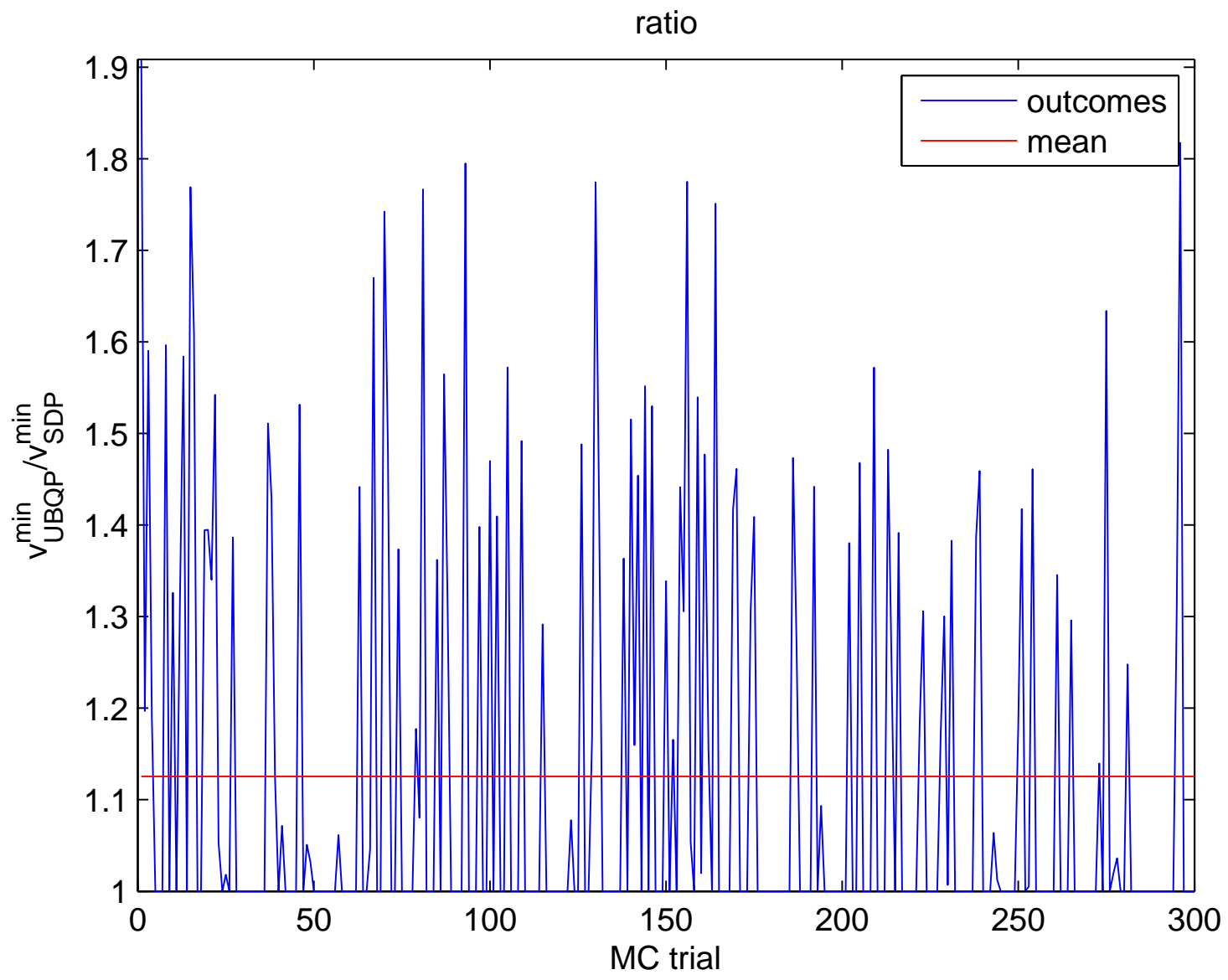

Table 3 shows the average ratio (mean) of $v_{\mathrm{UBQP}}^{\min } / v_{\mathrm{SDP}}^{\min }$ over 300 independent realizations of i.i.d. real-valued Gaussian $\mathbf{h}_{i},(i=1, \cdots, M)$ for several combinations of $M$ and $N$. The minimum value $(\min )$, the maximum value $(\max )$, the average value (mean) and the standard deviation (Std) of $v_{\mathrm{UBQP}}^{\min } / v_{\mathrm{SDP}}^{\min }$ over 300 independent realizations are also shown in Table 3. Table 4 shows the corresponding minimun value, maximum value, average value, and the standard deviation of $v_{\mathrm{UBQP}}^{\min } / v_{\mathrm{SDP}}^{\min }$ for $\mathbb{F}=\mathbb{C}$. We can see the results in both tables are significantly better than what is predicted by our worst-case analysis. In all test examples, the average values of $v_{\mathrm{UBQP}}^{\min } / v_{\mathrm{SDP}}^{\min }$ are lower than 3 (resp. lower than 2) when $\mathbb{F}=\mathbb{R}($ resp. when $\mathbb{F}=\mathbb{C}$ ). Moreover, the minimum value of $v_{\mathrm{UBQP}}^{\min } / v_{\mathrm{SDP}}^{\min }$ are exactly equal to 1 in most cases in both real and complex cases, which means the optimal solutions are obtained by our algorithm for some cases.

Figure 1 plots $v_{\mathrm{UBQP}}^{\min } / v_{\mathrm{SDP}}^{\min }$ for 300 independent realizations of i.i.d. real valued Gaussian $\mathbf{h}_{i}(i=$ $1, \cdots, M)$ for $M=5$ and $N=4$. Figure 2 shows the corresponding histogram. Figure 3 and Figure 4 show the corresponding results for i.i.d complex-valued circular Gaussian $\mathbf{h}_{i}(i=1, \cdots, M)$. Both the mean and the maximum of the upper bound $v_{\mathrm{UBQP}}^{\min } / v_{\mathrm{SDP}}^{\min }$ are lower in the complex case. 
Figure 4 Histogram of the outcomes in Figure 3 .

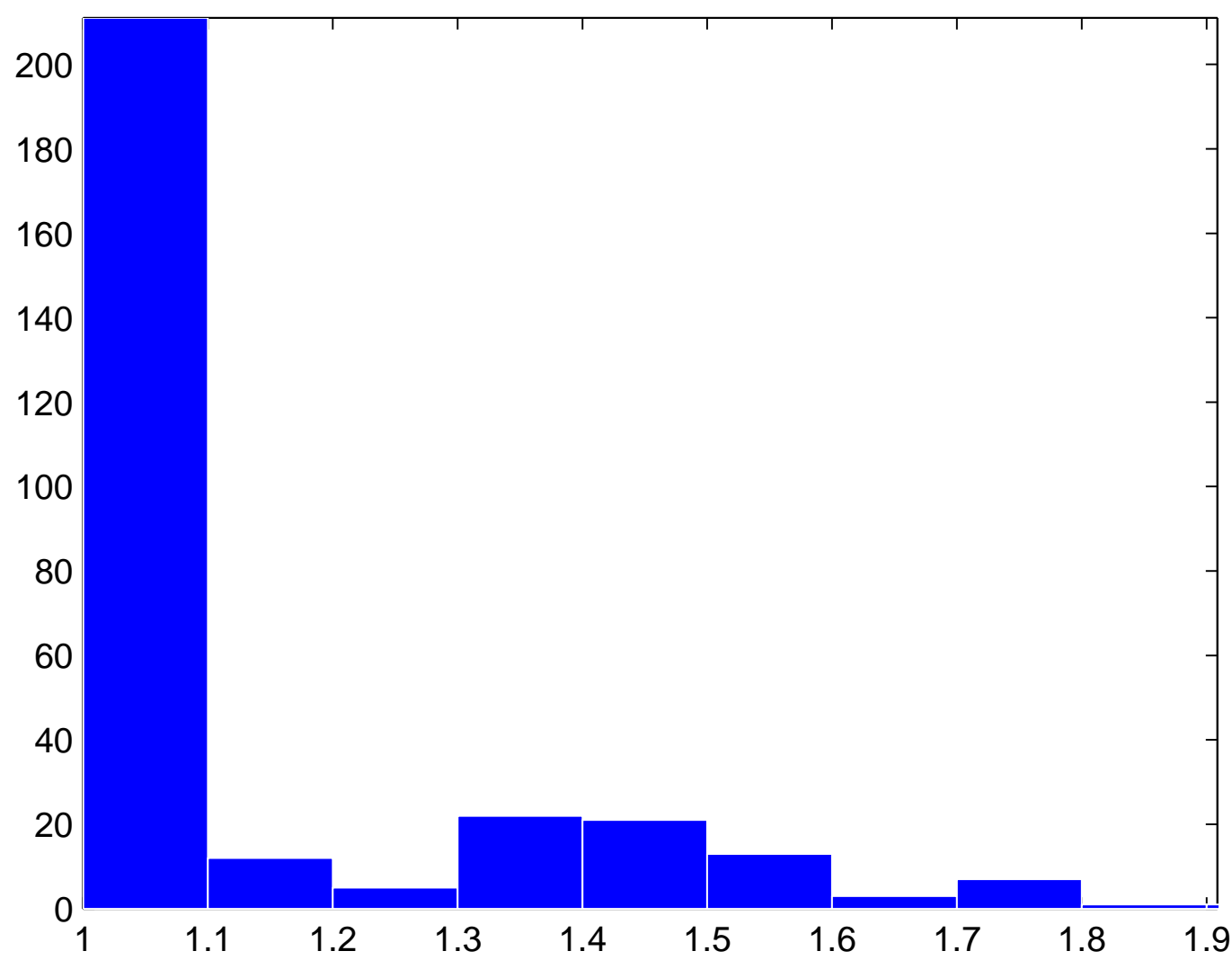

Moreover, our numerical results also corroborates well with our theoretic analysis. First, the upper bound of the approximation ratio is independent of the dimension of $\mathbf{w}$ : the results vary only slightly for $N=4, N=6$ and $N=8$ in both real and complex case. Second, from Table 3 and Table 4 , it can be shown that for fixed $N$, the maximum value and the average value of $v_{\mathrm{UBQP}}^{\min } / v_{\mathrm{SDP}}^{\min }$ over 300 independent trials grow as $M$ increases in all test examples except the case that $M=10$ and $N=8$ in Table 3. It corresponds to the result in Theorem 1 .

\section{Conclusion and Discussion}

In this paper, two classes of nonconvex quadratic optimization problems with mixed binary and continuous variables are considered, both of which are motivated by important applications in wireless networks. New SDP relaxation techniques with novel randomization algorithms are provided for these problems. It is shown that these efficient techniques can provide high quality approximate solutions. Our theoretic analysis provides useful insights on the effectiveness of the new SDP relaxation techniques for these two classes of MBQCQP problems. For both problems, finite and 
data independent approximation ratios are guaranteed. It should be pointed out that our worstcase analysis of SDP relaxation performance is based on certain special structure of the discrete and continuous variables. Using a counter example, we have shown in Xu et al. (2013) that SDP relaxation techniques and the corresponding analysis cannot extend directly to general MBQCQP problems.

\section{Acknowledgments}

This research is supported by the Chinese NSF under the grant 11101261 and the First-class Discipline of Universities in Shanghai.

\section{References}

Beck A, Teboulle M (2009) A convex optimization approach for minimizing the ratio of indefinite quadratic functions over an ellipsoid, Math. Program., 118:13-35.

Ben-Tal A, Nemirovski A, Roos C (2002) Robust solutions of uncertain quadratic and conic-quadratic problems, SIAM J. Optim., 13: 535-560.

Billionnet A, Elloumi B, Lambert A (2012) Extending the QCR method to general mixed-integer programs, Math. Program., 131:381-401.

Billionnet A, Elloumi S, Lambert A (2012) Convex reformulations of integer quadratically constrained problems, Talk presented at the In ternational Symposium on Mathematical Programming (ISMP).

Billionnet A, Elloumi S, Plateau M (2009) Improving the performance of standard solvers for quadratic 0-1 programs by a tight convex reformulation: the QCR method, Discr. Appl. Math., 157:1185-1197.

Burer S, Saxena A (2011) The MILP road to MIQCP. In: J. Lee and S. Leyffer (eds.) Mixed-Integer Nonlinear Programming, pp. 373-406, IMA Volumes in Mathematics and its Applications Vol.154, (Berlin, Springer).

Burer S, Letchford A(2012) Non-convex mixed-integer nonlinear programming: A survey, Surveys in Operations Research and Management Science, 17(2): 97-106.

Chang TH, Luo ZQ, Chi CY(2008) Approximation bounds for semidefinite relaxation of max-min-fair multicast transmit beamforming problem, IEEE Transactions on Signal Processing, 56:3932-3943.

Duran MA, Grossmann IE(1986) An outer-approximation algorithm for a class of mixed-integer nonlinear programs, Math. Program., 36:307-339.

Frieze AM, Jerrum M(1995) Improved approximation algorithms for max k-cut and max bisection, in Proceedings of the 4 th International IPCO Conference on Integer Programming and Combinatorial Optimization, pp. 1-13, (London, Springer-Verlag).

Goemans MX, Williamson DP(1995) Improved approximation algorithms for maximum cut and satisfiability problems using semidefinite programming, Journal of ACM, 42:1115-1145. 
Grant M, Boyd S(2011) CVX: Matlab software for disciplined convex programming, version 1.21, Apr. 2011.

He S, Luo ZQ, Nie J, Zhang S(2008) Semidefinite relaxation bounds for indefinite homogneous quadratic optimization, SIAM J. Optim., 19:503-523.

Hemmecke R, Koppe M, Lee J, Weismantel R(2010) Nonlinear integer programming, In M. Junger et al (eds.) 50 Years of Integer Programming 1958-2008, pp. 561-618, (Berlin, Springer).

Hong M, Xu M, Razaviyayn M, Luo ZQ(2013) Joint user grouping and linear virtual beamforming: Complexity, algorithms and approximation bounds, IEEE Journal on Selected Areas in Communications, Special issue on Virtual Multiantenna Systems, 31:2013-2027.

Huang Y, Zhang S, Complex matrix decomposition and quadratic programming, Math. Oper. Res., 32:758768.

Koppe M(2011), On the complexity of nonlinear mixed integer optimization, In J. Lee and S. Leyffer (eds.) Mixed-integer Nonlinear Programming, pp. 533-558. IMA Volumes in Mathematics and its Applications, Vol. 154 (Berlin, Springer).

Luo ZQ, Sidiropoulos ND, Tseng P, Zhang S(2007) Approximation bounds for quadratic optimization with homogeneous quadratic constraints, SIAM J. Optim., 18:1-28.

Luo ZQ, Ma Wk, So A.M.-C., Ye Y, Zhang S(2010) Semidefinite Relaxation of Quadratic Optimization Problems, IEEE Signal Processing Magazine, 27:20-34.

Nemirovski A, Roos C, Terlaky T(1999) On maximization of quadratic form over intersection of ellipsoids with common center, Math. Program., 86:463-473.

Ntranos V, Sidiropoulos ND, Tassiulas L(2009) On multicast beamforming for minimum outag, IEEE Transactions on Wireless Communications, 8:3172-3181.

Pataki G(1998) On the rank of extreme matrices in semidefinite programs and the multiplicity of optimal eigenvalues, Math. oper. Res., 23:339-358.

Saxena A, Bonami P, Lee J(2010) Convex relaxations of non-convex mixed integer quadratically constrained programs: extended formulations, Math. Program., 124:383-411.

Saxena A, Bonami P, Lee J(2011) Convex relaxations of non-convex mixed integer quadratically constrained programs: projected formulations, Math. Program., 130:359-413.

Sidiropoulos ND, Davidson TN, Luo ZQ(2006) Transmit beamforming for physical-layer multicasting, IEEE Transactions on Signal Processing, 54:2239-2251.

So A. M.-C, Ye Y, Zhang J(2008) A unified theorem on sdp rank reduction, Mathematics of Operations Research, 33:910-920.

Stubbs RA, Mehrotra S(1999) A branch-and-cut method for 0-1 mixed convex programming, Math. Program., $86: 515-532$. 

Article submitted to ; manuscript no. (Please, provide the manuscript number!)

Westerlund T, Pettersson F(1995) A cutting plane method for solving convex MINLP problems, Comput. and Chem. Eng., 19:131-136.

Xu Z, Hong M, Luo ZQ(2013) Semidefinite approximation for mixed binary quadratically constrained quadratic programs, submitted to SIAM J. Optim, available at arXiv:1302.4527.

Ye Y(2011) A .699-approximation algorithm for max-bisection, Mathematical Programming, 90:101-111.

Zhang S(2000) Quadratic maximization and semidefinite relaxation, Mathematical Programming, 87:453-465.

Zhang S, Huang Y(2004) Complex quadratic optimization and semidefinite programming, SIAM Journal on Optimization, 16:871-890. 